\title{
DETECÇ̃̃O E DIAGNÓSTICO DE FALHAS EM TROCADORES DE CALOR CASCO E TUBOS
}

\author{
F. C. CAMPANHA ${ }^{1}$, L. A. F. OLIVEIRA ${ }^{1}$, M. O. AGUIAR ${ }^{3}$, E. C. FURTADO ${ }^{2}$, G. M. \\ ALMEIDA $^{3}$ \\ ${ }^{1}$ Universidade Federal de São João del-Rei, Departamento de Engenharia Química e \\ Estatística \\ ${ }^{2}$ Universidade Federal de São João del-Rei, Departamento das Engenharias de \\ Telecomunicações e de Mecatrônica \\ ${ }^{3}$ Universidade Federal de Minas Gerais, Departamento de Engenharia Química \\ E-mail para contato: galmeida@deq.ufmg.br
}

\begin{abstract}
RESUMO - A exigência por processos mais seguros, econômicos e limpos é crescente, dada a maior pressão da sociedade, do mercado, dos governos e de associações, atualmente. Nesse cenário de restrições crescentes, o monitoramento das operações-chave nas indústrias químicas é essencial. Nesse trabalho, investigaram-se falhas usuais, abruptas e incipientes, em sensores e parâmetros, e de processo. Empregou-se o trocador de calor do tipo casco e tubos como estudo de caso. Após a criação de um benchmark, a geração de dados característicos de operações normal e de falhas, e a identificação de um sistema neural de monitoramento de falhas, observou-se a sua capacidade de detecção e de diagnóstico para falhas conhecidas, e de reconhecimento de uma situação como anormal no caso de falha desconhecida, uma informação útil como sinal de alerta.
\end{abstract}

\section{INTRODUÇÃO}

O monitoramento de operações-chave em indústrias químicas tem o papel de garantir segurança operacional e produção mais econômica e limpa. Com o aumento da pressão de associações, do governo e da sociedade, observou-se nas últimas duas décadas, tanto na academia quanto no meio industrial, o crescimento do espaço dedicado a área de monitoramento de processos químicos.

Uma atividade de monitoramento é composta pelas tarefas de detecção de falhas, com o objetivo de reconhecer desvios em relação à condição operacional normal, e de diagnóstico de falhas, com o objetivo de identificar as suas causas. Uma falha pode ser abrupta, a partir de um sinal degrau, ou incipiente, com um sinal de magnitude crescente ao longo do tempo. $\mathrm{O}$ segundo tipo é de maior interesse, uma vez que é a maneira usual de ocorrência de falhas nos processos industriais. A detecção e o diagnóstico de falhas incipientes, em relação às falhas abruptas, é geralmente mais difícil, devido à baixa magnitude dos sinais das variáveis de processo em seu estágio inicial. Investigaram-se ambos os tipos de falhas neste trabalho. Dada a percepção limitada do ser humano para sinais de baixa magnitude, o uso de sistemas computacionais de suporte à decisão como auxílio ao reconhecimento de operações com falhas é mandatório. Na prática, a construção de sistemas de monitoramento, automáticos, confiáveis e com resposta de tempo adequado, ainda é um desafio, em função da 
complexidade inerente às operações industriais, sendo, em geral, multivariáveis, não-lineares, ruidosos e de conhecimento parcial (Venkatasubramanian et al., 2003). Um sistema de monitoramento requer a existência de um modelo satisfatório do processo de interesse, cuja complexidade constitui-se em um fator limitante à construção de modelos fenomenológicos. Com o acúmulo significativo de dados históricos, observa-se, de modo crescente, a identificação de modelos sobre as operações industriais, diretamente a partir de dados históricos de processo (Chiang et al., 2001). Nesse trabalho, o processo químico de interesse é o trocador de calor do tipo casco e tubos. Comum a qualquer indústria química em aplicações variadas, esse equipamento tem a função de aquecer ou resfriar correntes de processo ao longo de toda a planta (Kreith e Bohn, 2003). Para o sistema de detecção e de diagnóstico de falhas, empregou-se um modelo de rede neural artificial, técnica pertencente a área de inteligência computacional (Haykin, 1998).

\section{OBJETIVO}

O objetivo geral é construir um sistema de detecção e de diagnóstico de falhas, abruptas e incipientes, para trocadores de calor casco e tubos. As falhas envolvem desvios em um sensor de uma malha de controle, em uma carga do processo, e em um de seus parâmetroschave. A geração dos bancos de dados de operação normal e de falhas é após a etapa inicial de implementação computacional do modelo do trocador de calor e dos cenários de falhas. $\mathrm{O}$ sistema de detecção e de diagnóstico de falhas é baseado em uma rede neural artificial.

\section{METODOLOGIA}

Apresenta-se, a seguir, a metodologia em conjunto com os resultados de cada uma das três etapas (objetivos específicos), necessárias para se realizar o objetivo geral desse trabalho, cujos resultados e discussão são apresentados na seção 4.

\subsection{Construção de um benchmark de um trocador de calor}

Inicialmente, implementou-se o modelo do trocador de calor casco e tubos, em malha fechada, disponível em Garcia (2005), no ambiente MATLAB/SIMULINK, um ambiente gráfico para simulações de sistemas dinâmicos (Figura 1). O modelo é um sistema de duas equações diferenciais ordinárias, em que $Q=$ vazão $\left(\mathrm{m}^{3} / \mathrm{h}\right), T=\operatorname{temperatura}\left({ }^{\circ} \mathrm{C}\right), \rho_{t, e}$ e $\rho_{t}=$ densidades da água quente de entrada $\left(998,21 \mathrm{~kg} / \mathrm{m}^{3}\right)$ e nos tubos $\left(995,65 \mathrm{~kg} / \mathrm{m}^{3}\right)$, respectivamente; $\rho_{c, e}$ e $\rho_{c}=$ densidades da água fria de entrada $\left(965,31 \mathrm{~kg} / \mathrm{m}^{3}\right)$ e no casco $\left(971,79 \mathrm{~kg} / \mathrm{m}^{3}\right)$, respectivamente; $V_{t}=$ volume externo dos tubos $\left(3,385 \cdot 10^{-3} \mathrm{~m}^{3}\right)$ e $V_{c}=$ volume interno disponível do casco $\left(4,557 \cdot 10^{-3} \mathrm{~m}^{3}\right) ; c_{p, a}=$ calor específico da água $(1,0$ $\left.\mathrm{kcal} /\left(\mathrm{kg} \cdot{ }^{\circ} \mathrm{C}\right)\right) ; U=$ coeficiente global de transferência de calor $\left(1.089,7 \mathrm{~W} /\left(\mathrm{m}^{2} \cdot \mathrm{K}\right)\right) ; A=$ área média de troca térmica $\left(0,8822 \quad \mathrm{~m}^{2}\right) ; \quad \mathrm{e}$ $\Delta T_{c t}=\left(\Delta T_{\text {max }, c t}-\Delta T_{\text {min }, c t}\right) / \ln \left(\Delta T_{\text {max }, c t} / \Delta T_{\text {min }, c t}\right)=$ média logarítmica da diferença de temperatura, com $\Delta T_{\max , c t}=T_{c, s}-T_{t, e}$ e $\Delta T_{\min , c t}=T_{c, e}-T_{t, s}$. Na sequência, definiram-se uma razão sinal-ruído (gaussiano) de 25 , um intervalo regular de amostragem, os cenários de falhas (conforme seção 3.2), um conjunto de seis variáveis de monitoramento, entre vazões e temperaturas, o sinal de saída do controlador PI e o deslocamento da haste da válvula de controle, entre outras características. Implementaram-se falhas em sensores e parâmetroschave e de processo, de natureza abrupta e incipiente, com sinais de magnitudes variadas. A 
Figura 1 é um esquema do resultado dessa etapa: um benchmark para a geração de dados de condições operacionais normais e de falha (seção 3.2) e para a identificação (seção 3.3) e análise de desempenho (seção 4) do sistema de detecção e de diagnóstico de falhas.

$$
\begin{array}{ll}
\frac{d\left(T_{t, s}\right)}{d t}=\frac{\rho_{t, e} \cdot Q_{t, e} \cdot c_{p, a} \cdot\left(T_{t, e}-T_{t, s}\right)+U \cdot A \cdot \Delta T_{c t}}{\rho_{t} \cdot V_{t} \cdot c_{p, a}}, & T_{t, s}(0)=40^{\circ} \mathrm{C} \\
\frac{d\left(T_{c, s}\right)}{d t}=\frac{\rho_{c, e} \cdot Q_{c, e} \cdot c_{p, a} \cdot\left(T_{c, e}-T_{c, s}\right)+U \cdot A \cdot \Delta T_{c t}}{\rho_{c} \cdot V_{c} \cdot C_{p, a}}, & T_{c, S}(0)=74,4^{o} C
\end{array}
$$

Figura 1 - Benchmark do trocador de calor em ambiente MATLAB/SIMULINK

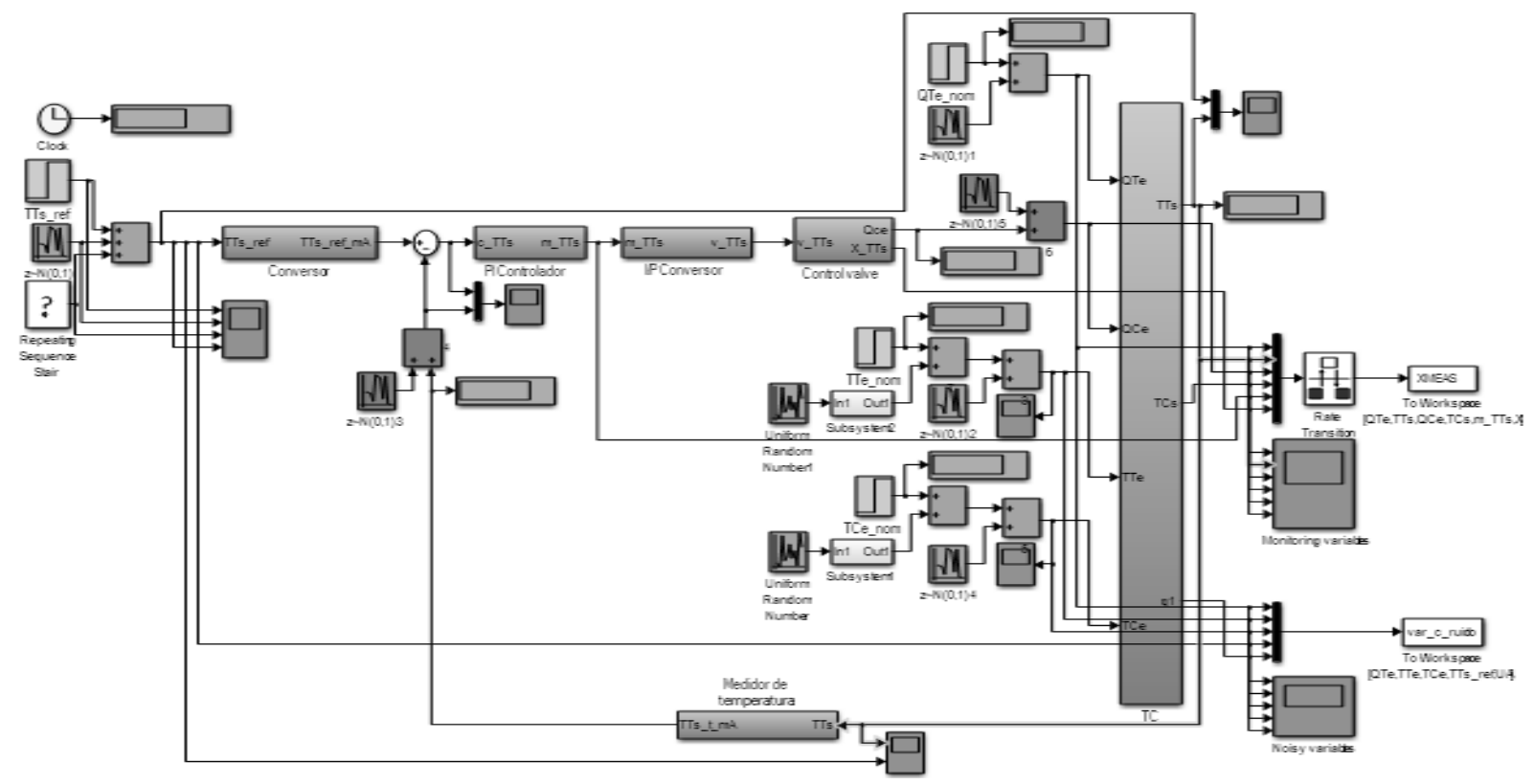

\subsection{Geração de cenários de operação normal e de falhas}

Após a seleção de um conjunto de seis variáveis de monitoramento, entre PVs, CVs, $\mathrm{MVs}^{1}$, e do sistema de controle regulatório, geraram-se bancos de dados, de treinamento e de teste, característicos de operações normais e de cenários de falhas, conforme a Figura 2. Neste trabalho, investigaram-se três falhas usuais em sistemas de trocadores de calor industriais (Utne et al., 2012; Narasimhan et al., 2008; Kesavan e Lee, 2001).

(1) Falha abrupta em sensor. Erro sistemático (bias) de 5\% (em relação à faixa de operação do dispositivo) na medição da temperatura do fluido de saída (variável controlada).

(2) Falha abrupta de processo. Perturbação degrau de 5\% (do valor nominal da variável) na temperatura de entrada do fluido quente.

(3) Falha incipiente em parâmetro. Distúrbio com taxa de decréscimo de $0,01 \%$ (em relação ao valor nominal do parâmetro), a cada instante de amostragem, no coeficiente global de transferência de calor.

\footnotetext{
${ }^{1}$ PVs: Variáveis de processo, CVs: Variáveis controladas, e MVs: Variáveis manipuladas.
} 
Figura 2 - Comportamento das variáveis de monitoramento: X: Deslocamento da haste da válvula (p.u., $[0,1]), C V$ : Sinal de saída do controlador PI (mA), T1: Temperatura de saída do

fluido quente do casco $\left({ }^{\circ} \mathrm{C}\right), \mathrm{F} 1$ : Vazão de entrada do fluido quente no casco $\left(\mathrm{m}^{3} / \mathrm{s}\right), \mathrm{T} 2$ :

Temperatura de saída do fluido frio nos tubos $\left({ }^{\circ} \mathrm{C}\right)$, e F2: Vazão de entrada do fluido frio nos tubos $\left(\mathrm{m}^{3} / \mathrm{s}\right)$, em função do tempo $(\mathrm{t})$; ocorrência da falha em $\mathrm{t}=1250$ ut (unidades de tempo).

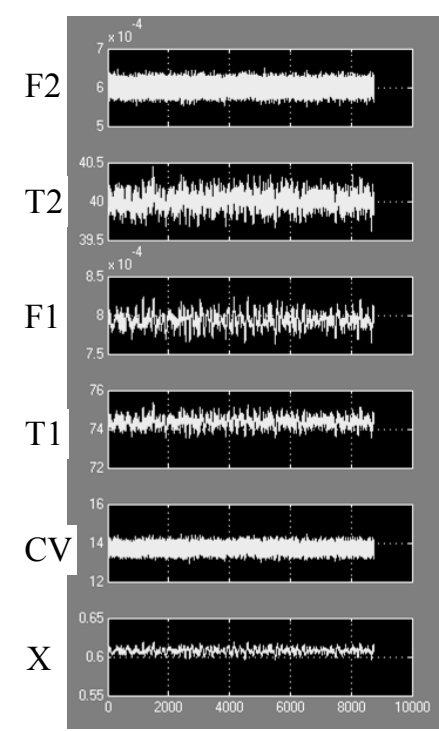

(a) Operação normal

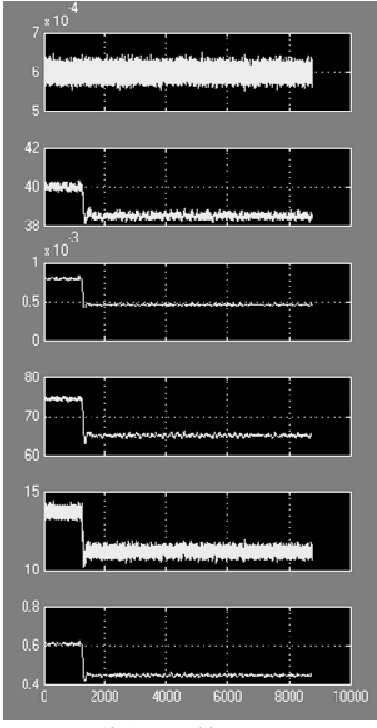

(b) Falha 1 (sensor)

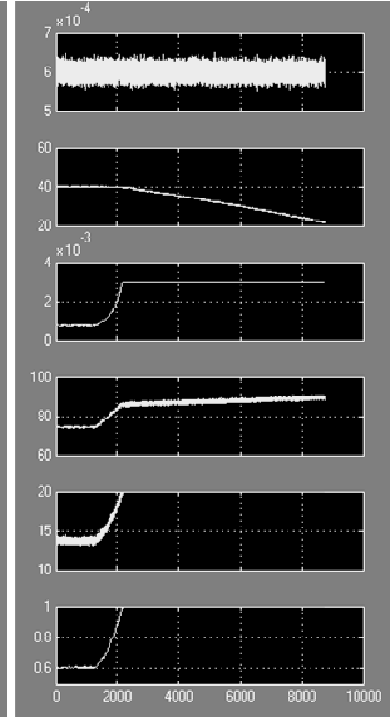

(c) Falha 2

(processo)

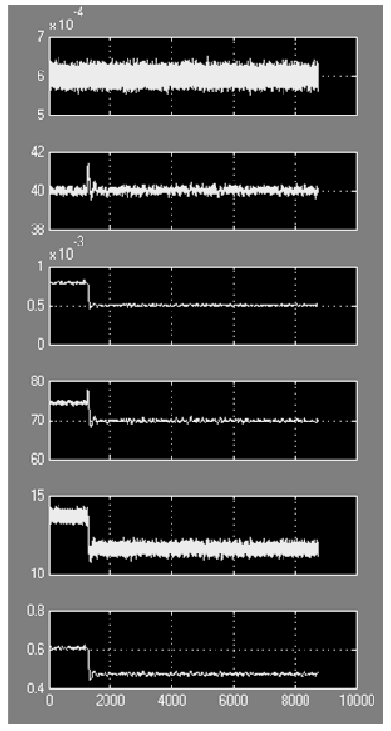

(d) Falha 3

(parâmetro)

\subsection{Identificação de um sistema de detecção e de diagnóstico de falhas}

Empregaram-se os conjuntos de dados de treinamento, característicos de operação normal e das falhas 1 e 2 (Figura 2(a-c)), para a identificação de um modelo neural, a ser usado como sistema de detecção e de diagnóstico de falhas. Adotou-se uma rede neural MLP (MultiLayer Perceptron), usual em aplicações de Engenharia, com funções tangente hiperbólica e identidade nos neurônios das camadas oculta e de saída, respectivamente. Utilizou-se a rede neural como um reconhecedor de padrões, ao se adotar a abordagem de problema de classificação. A saída do modelo neural é um vetor de três elementos binários (Duda et al., 2001), ou seja, o vetor [ $\left[\begin{array}{lll}1 & 0 & 0\end{array}\right]$ é indicativo de operação normal, [ $\left.\begin{array}{llll}0 & 1 & 0\end{array}\right]$, da falha 1 (sensor), e $\left[\begin{array}{lll}0 & 0 & 1\end{array}\right]$, da falha 2 (processo). Dada a sua natureza incipiente, não utilizouse a falha 3 (parâmetro). Adotou-se o procedimento hold out de estimação de parâmetros (matrizes de pesos), com $70 \%$ das observações para treinamento e o restante para validação, e como critério de seleção, o coeficiente de correlação linear $(r)$. O modelo neural final contém dez neurônios ocultos, cujo índice global de acertos de $100 \%$, dado o conjunto de validação.

\section{APRESENTAÇÃO E DISCUSSÃO DE RESULTADOS}

Em seguida às etapas anteriores (seção 3), submeteu-se o modelo neural final, ou seja, o sistema de detecção e de diagnóstico de falhas (SDD), aos cenários de condição normal e de falhas (Figura 2), usando-se conjuntos (independentes) de dados de teste (seção 3.2). As suas possíveis saídas são: condição normal, falha 1 ou falha 2. A Figura 3 mostra os resultados. A linha tracejada indica o instante de ocorrência das falhas 1 e 2 (degrau em $t=1250$ ut, em que ut $=$ unidades de tempo); não há uma linha equivalente para a falha 3 , por não compor o 


\section{Congresso Brasileiro de Engenharia Química em Iniciação Científica UFSCar - São Carlos - SP 16 a 19 de Julho de 2017}

conjunto de dados de treinamento (seção 3.3). Para a condição normal (Figura 3(a)), observa-se o seu reconhecimento por completo (linha azul-condição normal, em torno de 1; e linhas verde-falha 1 e vermelha-falha 2 , em torno de zero). A taxa de alarmes falsos de $0 \%$ mantém-se sob os cenários de falhas 1,2 e 3. Para a falha 1 (Figura 3(b)), observa-se, após 41 ut (em t $=1291 \mathrm{ut}$ ), a primeira detecção de uma operação anormal (queda gradual da linha azul-condição normal). A detecção completa de anormalidade ocorre após 123 ut (em $\mathrm{t}=1373$ ut; ascensão definitiva da linha vermelha-falha 2 sobre a linha azul-condição normal); porém, com um diagnóstico incorreto de falha 2. Após 214 ut (em t $=1464$ ut), temse o diagnóstico correto de falha 1 (ascensão definitiva da linha verde-falha 1 sobre a linha vermelha-falha 2). Nesse caso, há uma coincidência entre o primeiro diagnóstico e o diagnóstico completo. Para a falha 2 (Figura 3(c)), observa-se a primeira detecção de operação anormal após 196 ut (em t $=1446$ ut; queda gradual da linha azul-condição normal). A detecção completa de anormalidade ocorre após 277 ut (em t $=1527$ ut; ascensão definitiva da linha vermelha-falha 2 sobre a linha azul-condição normal). Tem-se, nesse mesmo instante de tempo, o primeiro diagnóstico correto de falha 2 . O diagnóstico completo de falha 2 ocorre após 525 ut (em $\mathrm{t}=1775$ ut; ascensão definitiva da linha vermelha-falha 1 sobre a linha verde-falha 1). Nesse caso, pode-se assumir que o reconhecimento da falha 2 ocorreu, de modo simultâneo, com a deteç̧ão completa de operação anormal. Para a falha 3 (Figura 3(d)), não utilizada no conjunto de treinamento do modelo neural, observou-se a capacidade do sistema em detectar uma condição operacional diferente daquelas já conhecidas, a saber, normal, falha 1 e falha 2. Isso porque, após 373 ut (em t $=1623$ ut), não há mais o reconhecimento de condição normal (queda relativa da linha azul-condição normal, em comparação com a Figura 3(a)), e nem de falha 1 ou de falha 2 (não ascensão das linhas verde-falha 1 e vermelha-falha 2). Tal sinal de alerta já é uma informação de grande valia; na prática, não é possível conhecer, a priori, o conjunto completo de falhas. A Tabela 1 é um resumo quantitativo dessa análise gráfica. Ressaltam-se, a robustez do sistema em relação a geração de alarmes falsos, a sua capacidade de detecção e de diagnóstico das falhas 1 (melhor resultado) e 2, e de reconhecimento de uma condição anormal desconhecida (falha 3 ).

Figura 3 - Resultados do sistema de detecção e de diagnóstico de falhas (SDD)

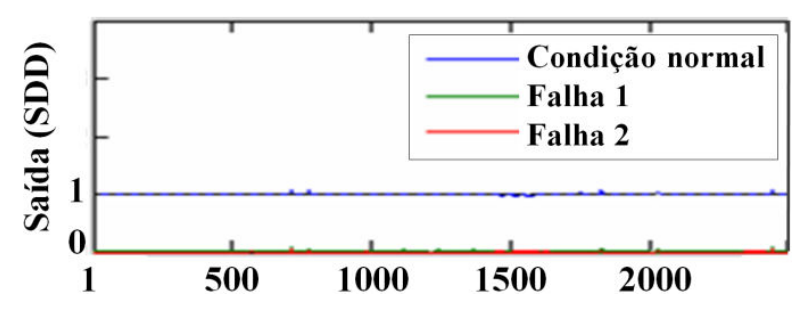

(a) Sob condição normal

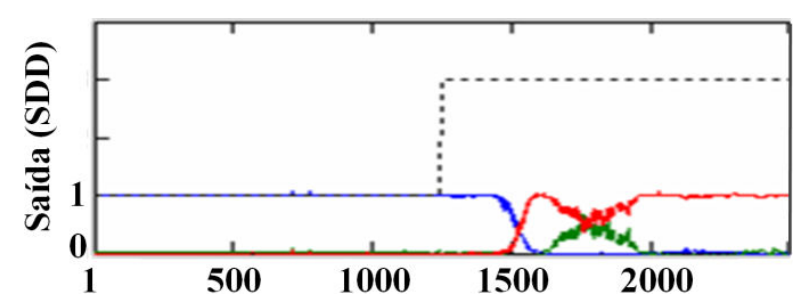

(c) Sob falha 2 abrupta (processo)

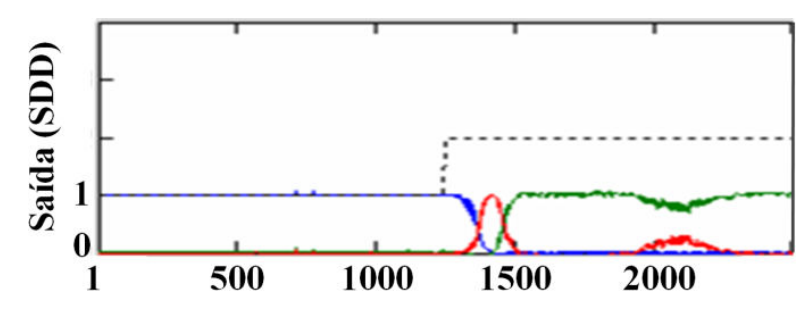

(b) Sob falha 1 abrupta (sensor)

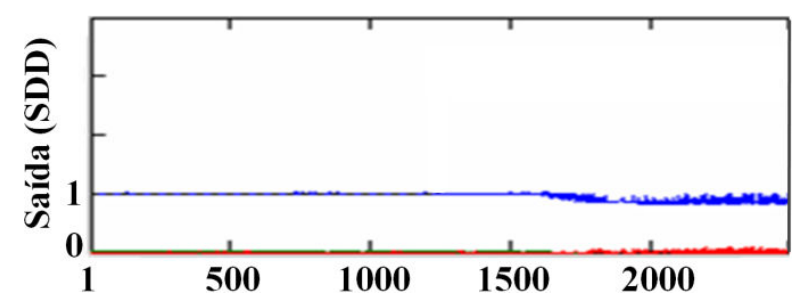

(d) Sob falha 3 incipiente (parâmetro) 
Tabela 1 - Resultados do sistema de detecção e de diagnóstico de falhas (SDD)

\begin{tabular}{|c|c|c|c|c|}
\hline Métrica de monitoramento & $\begin{array}{c}\text { Condição } \\
\text { normal }\end{array}$ & $\begin{array}{l}\text { Falha } 1 \\
\text { (sensor) }\end{array}$ & $\begin{array}{c}\text { Falha } 2 \\
\text { (processo) }\end{array}$ & $\begin{array}{c}\text { Falha } 3^{*} \\
\text { (parâmetro) }\end{array}$ \\
\hline Taxa de alarmes falsos & $0 \%$ & $0 \%$ & $0 \%$ & $0 \%$ \\
\hline Tempo até a $1^{\text {a }}$ detecção & & $41 \mathrm{ut}^{\dagger}$ & 196 ut & \multirow{2}{*}{373 ut } \\
\hline Tempo até a detecção completa & - & 123 ut & 277 ut & \\
\hline Tempo até o $1^{0}$ diagnóstico & - & 214 ut & 277 ut & \multirow{2}{*}{$\begin{array}{l}\text { O sistema é capaz de reconhecer } \\
\text { uma condição diferente daquelas já } \\
\text { conhecidas: normal e falhas } 1 \text { e } 2 \text {. }\end{array}$} \\
\hline $\begin{array}{l}\text { Tempo até o diagnóstico } \\
\text { completo }\end{array}$ & - & 214 ut & 525 ut & \\
\hline
\end{tabular}

†ut = Unidades de tempo; ${ }^{\star}$ Não utilizado na identificação do sistema de detecção e de diagnóstico de falhas (seção 3.3).

\section{CONSIDERAÇÕES FINAIS}

Um sistema de monitoramento, automático, confiável e com tempo de resposta adequado, ainda é um desafio. Neste trabalho de iniciação científica, exploraram-se falhas usuais ao monitoramento de processos químicos contínuos, a saber, em sensor, de processo e em parâmetro-chave, abruptas ou incipientes. Utilizou-se o modelo de um trocador de calor casco e tubos como estudo de caso e uma rede neural artificial como sistema de detecção e de diagnóstico de falhas. Ao final, observou-se um desempenho satisfatório, com capacidade de detecção e de diagnóstico, ou pelo menos de reconhecimento de uma condição anormal, informação já importante como sinal de alerta.

\section{REFERÊNCIAS BIBLIOGRÁFICAS}

CHIANG, L. H.; RUSSEL, E. L.; BRAATZ, R. D. Fault Detection and Diagnosis in Industrial Systems. Londres: Springer, 2001, 279p.

DUDA, R. O.; HART, P. E.; STORK, D. G. Pattern Classification. Nova Iorque: Wiley, 2001, 654p.

GARCIA, C. Modelagem e Simulação de Processos Industriais e de Sistemas Eletromecânicos. São Paulo: EDUSP, 2a ed., 2005, 688p.

HAYKIN, S. Neural Networks: A Comprehensive Foundation. Nova Iorque: Prentice Hall, 1998, 842p.

KESAVAN, P.; LEE, J. H. A set based approach to detection and isolation of faults in multivariable systems. Computers and Chemical Engineering. v. 25, n. 7-8, p. 925-940, 2001.

KREITH, F; BOHN, M. S. Princípios de Transferência de Calor. SP: Thomson, 2003, 623p.

NARASIMHAN, S.; VACHHANI, P.; RENGASWAMY, R. New nonlinear residual feedback observer for fault diagnosis in nonlinear systems, Automatica. v. 44, n. 9, p. 22222229, 2008.

UTNE, I. B.; BRUROK, T.; RØDSETH, H. A structured approach to improved condition monitoring. J. Loss Prevention in the Process Industries. v. 25, n. 3, p. 478-488, 2012.

VENKATASUBRAMANIAN, V.; RENGASWAMY, R.; YIN, K.; KAVURI, S. N. A Review of Process Fault Diagnosis - Part I: Quantitative Model-based Methods, Computers and Chemical Engineering. v. 27, n. 3, p. 293-311, 2003. 